\title{
Occurrence of COVID-19 in priority groups receiving ChAd0x1 nCoV-19 coronavirus vaccine (recombinant): a preliminary analysis from north India
}

\section{Upinder Kaur}

Institute of Medical Sciences, Banaras Hindu University

\section{Sapna Bala}

Banaras Hindu University

\section{Bisweswar Ojha}

Institute of Medical Sciences, Banaras Hindu University

\section{Sumit Jaiswal}

Institute of Medical Sciences, Banaras Hindu University

\section{Sangeeta Kansal}

Institute of Medical Sciences, Banaras Hindu University

Sankha Shubhra Chakrabarti ( $\nabla$ sankha.geriatrics@gmail.com )

Institute of Medical Sciences, Banaras Hindu University

\section{Short Report}

Keywords: Breakthrough, Delta, Real-world, Variants

Posted Date: August 2nd, 2021

DOI: https://doi.org/10.21203/rs.3.rs-772465/v1

License: (c) (1) This work is licensed under a Creative Commons Attribution 4.0 International License.

Read Full License

Version of Record: A version of this preprint was published at Journal of Medical Virology on September 12th, 2021. See the published version at https://doi.org/10.1002/jmv.27320. 


\section{Abstract}

Background: In randomized controlled settings, vaccine efficacy close to $70 \%$ against symptomatic COVID-19 has been demonstrated by the ChAdOx1 nCoV-19 vaccine which is a recombinant chimpanzee adenovirus based vaccine expressing the SARS-CoV-2 spike protein. Post approval studies are however necessary to validate the findings in the real world.

Methods: A prospective observational study is being conducted in a tertiary hospital of north India since $5^{\text {th }}$ February 2021 with the primary objective of determining safety of COVID-19 vaccines and the secondary objective of assessing the rate of occurrence of COVID-19 in vaccinated group. High risk group comprising health care workers, other frontline workers (police, sanitary workers etc) and elderly citizens who were initially focus groups for vaccine roll-out in India, were enrolled in the study. The study included all vaccine recipients who provided consent and were enrolled at the time of receiving the first or second dose of COVISHIELD vaccine, and followed up telephonically.

Results: Among 1650 enrolled vaccine recipients, 1500 participants of the study (Female/Male: 472/1028; mean age 38.8 years) completed at least 2 months of follow-up, after the second dose. The common comorbidities in study participants were hypertension (170, 11.3\%), diabetes (142, 9.5\%), and hypothyroidism $(54,3.6 \%)$. Of those who received a single dose of vaccine $(n=65)$, laboratory confirmed SARS-CoV-2 infection was observed in 27 individuals (41.5\%) and 3 were suspects. Severity wise, infections were mild in 21 out of $30(70 \%)$ cases, moderate in five (16.7\%) and severe in two (6.7\%). Of those who received both doses of vaccine $(n=1435), 388$ were diagnosed as confirmed or suspect cases of SARS-CoV-2 infection. Of these 388, RT-PCR positivity was seen in 271 (18.9\%) individuals, $82(5.7 \%)$ were labelled as 'suspects' and 35 (2.4\%) were RT-PCR negative suspects. Severity wise, majority of SARSCoV-2 infections were 'mild' (331/388, 85.3\%), followed by 'moderate' (33/388, 8.5\%) and 'severe' (6/388, $1.5 \%$ ). 404 out of the 1500 total participants were doctors including consultant/teaching faculty, resident doctors, and those in general practice. Among the 377 doctors who received both doses of vaccine, 160 were diagnosed as confirmed or suspect cases of SARS-CoV-2 infection. Of these, 131 (34.7\%), 17 (4.5\%) and $12(3.2 \%)$ were laboratory confirmed cases, 'suspects' and RT-PCR negative suspects respectively. The infection was asymptomatic, 'mild', 'moderate' and 'severe' in 9 (5.6\%), 130 (81.3\%), 16 (10\%) and 5 (3.1\%) respectively. Breakthrough infections occurring at $>14$ days after receiving the second dose were seen in 148 doctors who received both doses (39.2\%), or 119 doctors (31.6\%) if only laboratory confirmed cases were considered. Four deaths occurred in the study participants during the study period, two in partially vaccinated group and two in fully vaccinated group. Two of these participants, both in partially vaccinated group had developed SARS-CoV-2 infection during their follow-up.

Conclusion: The disproportionately high occurrence of SARS-CoV-2 infection and COVID-19 in priority vaccinated groups in our study can be explained to some extent by the existence of variants such as the delta which might have escaped the vaccine generated immune protection. Despite the high incidence, the severity of COVID-19 was observed to be low. Since the ongoing study was primarily focused on adverse events following immunization (AEFIs) and enrolled only vaccinated individuals, the secondary outcome 
results lack a control unvaccinated group. However, the result of this preliminary analysis necessitates vigorous research on the performance of vaccines against variants, optimal timing of vaccination, need for boosters, and also optimal timings of effectiveness studies to guide future vaccination policy.

\section{Introduction}

To deal with the ongoing COVID-19 crisis, some countries have given emergency use authorization to COVID-19 vaccines. These include mRNA vaccines, adeno viral vector-based vaccines and inactivated SARS-CoV-2 vaccines. In randomized controlled settings, efficacy close to $70 \%$ against symptomatic COVID-19 has been demonstrated for the ChAdOx $1 \mathrm{nCoV}-19$ vaccine which uses a recombinant chimpanzee adenovirus coding for the SARS-CoV-2 spike protein. Post approval studies are however necessary to validate the findings in the real world. A prospective observational study is being conducted in a tertiary hospital of north India since 5th February 2021 with the primary objective of determining safety of COVID-19 vaccines and the secondary objective of assessing the rate of occurrence of COVID-19 in vaccinated group. High risk group comprising health care workers, other frontline workers (police, sanitary workers etc) and elderly citizens who were initially focus groups for vaccine roll-out in India, were enrolled in the study. As per government policies, COVISHIELD, based on the ChAdOx1 platform was the designated vaccine for the study centre during the study enrolment period. The authors recently demonstrated a favourable safety profile of COVISHIELD when assessed up to seven days after second dose.[1] Here we report the preliminary findings with respect to our secondary outcome measure of postvaccination COVID-19 or SARS-CoV-2 infections. The data is based on at least 2 months of follow-up since second dose of vaccine in enrolled participants who could be successfully contacted telephonically for details.

\section{Methods}

The study included all vaccine recipients who provided consent and were enrolled at the time of receiving the first or second dose of COVISHIELD vaccine. The recipients included predominantly medical and paramedical personnel as they were the initial beneficiaries of vaccine roll-out. In the later stages of enrolment, elderly non-healthcare workers who visited the study centre for vaccination, were also enrolled. Baseline demographic details of each participant was collected in a pre-designed case report form.[1] Enrolment was continued till target sample size $(n=1650)$ was reached. As part of the ongoing prospective observational study, each participant is being monitored telephonically at specific intervals following second dose, for a total duration of one year (study duration). Those who received only one dose are also being followed up in a similar fashion. For secondary objective, details pertaining to development of COVID-19 like symptoms, RT-PCR positivity, or rapid antigen test positivity for SARS-CoV-2 at any time following vaccination, were collected. Symptoms of COVID-19 if any, need for hospitalization, oxygen requirement details were also collected. In case of deaths of any of the vaccine recipients, telephonic ascertainment of cause of death was done from family members, and a request was made to provide 
medical certification documents. This interim analysis of the vaccine recipients is being performed after at least two months of follow up since second dose.

COVID-19 cases were defined as 'confirmed' and 'suspect' based on investigators' assessment and guided by recommendations issued by the Ministry of Health and Family Welfare, Government of India.[2] Any person with laboratory confirmation of SARS-CoV-2 infection irrespective of clinical signs or symptoms was considered a confirmed case. COVID-19 suspect cases are those having a suggestive clinical pattern of symptoms, and exposure to confirmed/probable cases of COVID-19 within the past 14 days. They were divided into COVID-19 suspects (Not tested)- in the absence of rapid antigen test or RT-PCR test, and RTPCR negative COVID-19 suspects- with a negative RT-PCR test.

\section{Results}

Among 1650 enrolled vaccine recipients, 1500 participants of the study (Female/Male: 472/1028) completed at least 2 months of follow-up, after the second dose. Baseline characteristics of the participants in this preliminary analysis are provided in Table 1. Among these 1500, 1435 participants received both doses and 65 received only one dose of the vaccine.

\section{Occurrence and severity of COVID-19 after single dose of vaccine:}

Of those who received a single dose of vaccine $(n=65)$, laboratory confirmed SARS-CoV-2 infection was observed in 27 individuals (41.5\%). Two individuals (3.1\%) were labelled as 'suspects', and one (1.5\%) as RT-PCR negative suspect. Severity wise, infections were mild in 21 out of $30(70 \%)$ cases, moderate in five $(16.7 \%)$ and severe in two (6.7\%). One participant (3.3\%) was asymptomatic. Three individuals required hospitalization ( $10 \%$ of cases) for oxygen requirement. Two deaths were observed in this group, one in an elderly male with pre-existing coronary artery disease who died because of unspecified cardiac event following recovery from COVID-19, and the other in an elderly male with comorbid diabetes and congestive heart failure who succumbed to unspecified post-COVID-19 complications. Details of both events were based on history provided by family members. (Table 1 )

\section{Occurrence and severity of COVID-19 after both doses of vaccine:}

Of those who received both doses of vaccine $(n=1435), 388$ were diagnosed as confirmed or suspect cases of SARS-CoV-2 infection. Of these 388, RT-PCR positivity was seen in 271 (18.9\%) individuals, 82 $(5.7 \%)$ were labelled as 'suspects' and $35(2.4 \%)$ were RT-PCR negative suspects. Severity wise, majority of SARS-CoV-2 infections were 'mild' (331/388, 85.3\%), followed by 'moderate' $(33 / 388,8.5 \%)$ and 'severe' $(6 / 388,1.5 \%)$. Infection was asymptomatic in 18/388 (4.7\%). Infection occurred within $\leq 14$ days of receiving the second dose in 31 and after 14 days of receiving second dose in 357. Breakthrough infection rate was determined to be close to $25 \%$ (357/1435), and $17 \%$ (246/1435) if only laboratory confirmed cases were considered. Nineteen individuals needed hospitalization (1.3\%) of whom six were admitted because of respiratory distress or oxygen requirements $(0.4 \%)$. Two deaths were reported. One was a 
middle-aged man with comorbid diabetes who developed cellulitis of the lower limb leading to sepsis and encephalopathy and died due to multiorgan failure. He was assessed twice for SARS-CoV-2 infection but tested negative. The other death was of a healthcare worker with comorbid diabetes and hypertension who died of an unspecified cardiac event. COVID-19 details for this participant could not be obtained from the family members. Details of both events were based on history provided by family members. (Table 1)

\section{Occurrence of COVID-19 in doctors:}

404 out of the 1500 total participants were doctors including consultant/teaching faculty, resident doctors, and those in general practice. Among these, 27 had received one dose and 377 had received both doses of the vaccine, at the time of analysis. Among the 27 having received a single dose, RT-PCR confirmed SARSCoV-2 infection occurred in 18 (66.7\%) and one participant was labelled as RT-PCR negative 'suspect'. Infection was 'asymptomatic' in one (5.3\%), and 'mild', 'moderate' and 'severe' in 14/19 (73.7\%), 3/19 (15.8\%) and $1 / 19(5.3 \%)$, respectively.

Among the 377 doctors who received both doses of vaccine, 160 were diagnosed as confirmed or suspect cases of SARS-CoV-2 infection. Of these, 131 (34.7\%), 17 (4.5\%) and 12 (3.2\%) were laboratory confirmed cases, 'suspects' and RT-PCR negative suspects respectively. The infection was asymptomatic, 'mild', 'moderate' and 'severe' in 9 (5.6\%), 130 (81.3\%), 16 (10\%) and 5 (3.1\%) respectively. Breakthrough infections occurring at $>14$ days after receiving the second dose were seen in 148 doctors (39.2\%), or 119 doctors (31.6\%), if only laboratory confirmed cases were considered. (Table 1)

\section{Discussion}

ChAdOx1 vaccine was found to reduce the rate of symptomatic COVID-19 by around $70 \%$ in randomized controlled trials and similar protection against symptomatic COVID-19 has been observed in some real world studies too.[3-5] The percentage of individuals developing COVID-19 in controlled and real-world settings after vaccination with ChAdOx1 vaccine has been low and has varied from $0.6-1.2 \% .[3,6]$ However, there may be differences in vaccine effectiveness and breakthrough infection rates depending on when surveillance is carried out with respect to the timing of COVID-19 waves. In a relatively quiescent period after the downslope of a wave, there may be few cases while the case numbers including breakthrough may be higher during an ongoing wave. Victor PJ et al, reported a close to $10 \%$ rate of occurrence of COVID-19 in fully vaccinated healthcare workers from a tertiary care hospital in southern India. Rates of hospitalization and oxygen requirement in the vaccinated group were $0.9 \%$ and $0.06 \%$ respectively. In our study, $4.6 \%$ of individuals who received one dose and $0.4 \%$ who received both doses, were hospitalized for oxygen needs. Some participants who developed hypoxemia preferred home based management including oxygen supplementation. Another Indian study from a tertiary hospital in northern India has reported a rather low rate (2.6\%) of occurrence of COVID-19 after two doses of COVISHIELD vaccine. Even after excluding RT-PCR negative and untested suspects, the rates of SARS-CoV-2 infection were much higher (close to 19\%) in the fully vaccinated participants in our study, and nearly $34 \%$ among fully vaccinated doctors. 
This disproportionately high occurrence of SARS-CoV-2 infection and COVID-19 in vaccinated high-risk groups in our study can be explained to some extent by the existence of variants such as the delta which might have escaped the vaccine generated immune protection. An outbreak of the delta variant of SARSCoV-2 was reported following the first dose of ChAdOx1 based vaccine (VAXZEVRIA) in an elderly care home in London.[7] A reduced protection against the delta variant compared to alpha variant has been shown for ChAdOx1 nCoV-19 vaccine recently.[8] Other potential explanations for the discrepancy in the rate of occurrence of SARS-CoV-2 infection between different Indian studies can be related to the study designs and regional variations. The study by Rana et al mentions the minimum follow up period of 2 weeks following second dose and also the median time to breakthrough following second dose (29.5 days) but does not mention the total duration of follow up. The possibility of infections happening in other healthcare workers with immunity waning with time, hence cannot be ruled out.[9] The south Indian study which reported relatively higher rate of infections coincided in timing with our study, and the peak of the second wave in India.[10] However, regional variations between different states may explain the difference in breakthrough rates. Further, there may be a difference in dominant SARS-CoV-2 variants between the two hospitals. We are continuing the follow up of all included participants and believe the future results to provide a more accurate representation of COVID-19 occurrence.

Despite the high rate of occurrence of SARS-CoV-2 infections in vaccinated individuals, the severity of COVID-19 was observed to be low. $41 \%$ individuals who received only one dose of the vaccine and $19 \%$ of those who received both doses developed laboratory confirmed SARS-CoV-2 infection. Occurrence of 'severe' COVID-19 was 7.7 times lower (0.4\%) in fully vaccinated participants compared to partially vaccinated group (3.1\%). These rates might reflect a dose response relationship of the vaccine in reducing infections as well as disease severity. Of the four deaths observed, one was possibly related to COVID-19 and occurred in a partially vaccinated elderly patient. No deaths due to COVID-19 were observed in fully vaccinated individuals.

Since the ongoing study was primarily focused on adverse events following immunization (AEFIs) and enrolled only vaccinated individuals, the secondary outcome results lack a control unvaccinated group. Hence, the authors cannot comment upon the effectiveness of vaccine against infection, hospitalization, and death. A separate study has been initiated to analyse the outcomes in both unvaccinated and vaccinated healthcare workers and auxiliary staff of the hospital.

\section{Conclusion}

The second wave of the COVID-19 pandemic hit large parts of India in April and May. The findings of the present study suggest that real-world protection by the ChAdOx 1 vaccine against symptomatic COVID-19 may not be as high as observed in clinical trial settings or even in real world settings when a wave has just subsided. Differences may also exist depending on the prevailing variants. Though the rate of infection in vaccinated individuals was more than 20 times higher than what has been observed in randomized controlled settings, severe COVID-19 occurred only in $0.4 \%$ fully vaccinated individuals. Detailed analysis of cause of death in the four cases with mortality could not be performed due to the observational nature 
of the study. The result of this preliminary analysis necessitates vigorous research on the performance of vaccines against variants, optimal timing of vaccination, need for boosters, and also optimal timings of effectiveness studies to guide future vaccination policy.

\section{Declarations}

Acknowledgements: None

Conflict of interest: None Funding: None

Ethical Permission: Ethical approval was obtained prior to conducting the study from the institute ethics committee of the Institute of Medical Sciences, Banaras Hindu University, Varanasi, UP, India. No human experimentation was performed. Written informed consent was taken from all participants of this observational study.

\section{References}

1. Kaur U, Ojha B, Pathak BK, Singh A, Giri KR, Singh A, et al. (2021). A prospective observational safety study on ChAdOx1 nCoV-19 corona virus vaccine (recombinant) use in healthcare workers- first results from India. EClinicalMedicine, 101038.

2. Ministry of Health and Family Welfare Government of India Updated Clinical Management Prootocol for COVID-19.

https://www.mohfw.gov.in/pdf/UpdatedClinicalManagementProtocolforCOVID19dated03072020.pdf. Accessed 20 Sep 2020.

3. Voysey M, Clemens SAC, Madhi SA, Weckx LY, Folegatti PM, Aley PK, et al. (2021). Safety and efficacy of the ChAdOx1 nCoV-19 vaccine (AZD1222) against SARS-CoV-2: an interim analysis of four randomised controlled trials in Brazil, South Africa, and the UK. Lancet, 397:99-111.

4. Lopez Bernal J, Andrews N, Gower C, Robertson C, Stowe J, Tessier E, et al. (2021). Effectiveness of the Pfizer-BioNTech and Oxford-AstraZeneca vaccines on covid-19 related symptoms, hospital admissions, and mortality in older adults in England: test negative case-control study. BMJ, n1088.

5. Shrotri M, Krutikov M, Palmer T, Giddings R, Azmi B, Subbarao S, et al. (2021). Vaccine effectiveness of the first dose of ChAdOx1 nCoV-19 and BNT162b2 against SARS-CoV-2 infection in residents of Long-Term Care Facilities (VIVALDI study). medRxiv, 2021.03.26.21254391.

6. Paris C, Perrin S, Hamonic S, Bourget B, Roué C, Brassard O, et al. (2021). Effectiveness of mRNABNT162b2, mRNA-1273, and ChAdOx1 nCoV-19 vaccines against COVID-19 in healthcare workers: an observational study using surveillance data. Clin Microbiol Infect. doi: 10.1016/j.cmi.2021.06.043.

7. Williams S V, Vusirikala A, Ladhani SN, Fernandez Ruiz De Olano E, lyanger N, Aiano F, et al. (2021). An outbreak caused by the SARS-CoV-2 Delta (B.1.617.2) variant in a care home after partial vaccination with a single dose of the COVID-19 vaccine Vaxzevria, London, England, April 2021. Eurosurveillance. doi: 10.2807/1560-7917.ES.2021.26.27.2100626. 
8. Lopez Bernal J, Andrews N, Gower C, Gallagher E, Simmons R, Thelwall S, et al. (2021). Effectiveness of Covid-19 Vaccines against the B.1.617.2 (Delta) Variant. N Engl J Med, NEJMoa2108891.

9. Rana K, Mohindra R, Pinnaka L (2021). Vaccine Breakthrough Infections with SARS-CoV-2 Variants. N Engl J Med, 385:e7.

10. Victor PJ, Mathews KP, Paul H, Murugesan M, Mammen JJ (2021). Protective Effect of COVID-19 Vaccine Among Health Care Workers During the Second Wave of the pandemic in India. Mayo Clin Proc. doi: 10.1016/j.mayocp.2021.06.003.

\section{Tables}




\begin{tabular}{|c|c|c|}
\hline \multicolumn{3}{|c|}{ Demographic characteristics of all vaccine recipients $(n=1500)$} \\
\hline Male, Female & 1028,472 & \\
\hline $\begin{array}{l}\text { Age in years } \\
(\text { Mean } \pm \mathrm{SD})\end{array}$ & $38.8 \pm 12.8$ & \\
\hline $\begin{array}{l}\text { Diabetes mellitus, } \mathrm{n} \\
(\%)\end{array}$ & $142(9.5)$ & \\
\hline Hypertension, n (\%) & $170(11.3)$ & \\
\hline Heart diseases, n (\%) & $\begin{array}{l}\text { CAD: } 21(1.4) \\
\text { RHD: } 1(0.1) \\
\text { ASD: } 1(0.1)\end{array}$ & \\
\hline Hypothyroidism, n (\%) & $54(3.6)$ & \\
\hline $\begin{array}{l}\text { Respiratory diseases, } \mathrm{n} \\
(\%)\end{array}$ & $\begin{array}{l}\text { Asthma: } 41 \text { (2.8) } \\
\text { ILD: } 1(0.1)\end{array}$ & \\
\hline \multicolumn{3}{|c|}{ Characteristics of COVID-19 $(n=1500)$} \\
\hline & $\begin{array}{l}\text { Participants who } \\
\text { received single } \\
\text { dose of vaccine } \\
(\mathrm{n}=65)\end{array}$ & $\begin{array}{l}\text { Participants who received } \\
\text { both doses of vaccine } \\
(n=1435)\end{array}$ \\
\hline $\begin{array}{l}\text { Confirmed or suspect } \\
\text { cases, } \mathrm{n}(\%)\end{array}$ & $30(46.1)$ & $\begin{array}{l}388(27) \\
\leq 14 \text { days after second dose: } \\
n=31 \\
>14 \text { days after second dose: } \\
n=357\end{array}$ \\
\hline $\begin{array}{l}\text { RT-PCR confirmed } \\
\text { SARS-CoV-2 infections } \\
\text { COVID-19 suspect (Not } \\
\text { tested)* } \\
\text { RT-PCR negative } \\
\text { COVID-19 suspect }{ }^{*}\end{array}$ & $\begin{array}{l}27(41.5) \\
2(3.1) \\
1(1.5)\end{array}$ & $\begin{array}{l}271(18.9) \\
82(5.7) \\
35(2.4)\end{array}$ \\
\hline $\begin{array}{l}\text { Severity }{ }^{\mathbb{I}} \text { (all confirmed } \\
\text { or suspect) } \\
\text { Asymptomatic } \\
\text { Mild } \\
\text { Moderate }\end{array}$ & $\begin{array}{l}1(3.3) \\
21(70) \\
5(16.7)\end{array}$ & $\begin{array}{l}18(4.6) \\
331(85.3) \\
33(8.5) \\
6(1.5)\end{array}$ \\
\hline
\end{tabular}




\begin{tabular}{|c|c|c|}
\hline $\begin{array}{l}\text { Severe } \\
\text { Unsure }\end{array}$ & $\begin{array}{l}2 \text { ( } 6.7) \text {, including } \\
\text { one death } \\
1 \text { (3.3), one death } \\
\text { (other cause) }\end{array}$ & -- \\
\hline Hospitalization needed & $\begin{array}{l}3 \text { (all for oxygen } \\
\text { requirement) }\end{array}$ & $\begin{array}{l}19 \text { (6 for oxygen } \\
\text { requirement, } 12 \text { for } \\
\text { observation, } 1 \text { for general } \\
\text { caregiver support) }\end{array}$ \\
\hline $\begin{array}{l}\text { Median time of onset } \\
\text { following vaccine dose } \\
\text { in days (Q1,Q3; range) }\end{array}$ & $45(29,55 ; 1-73)$ & $39(28,47 ; 0-99)$ \\
\hline $\begin{array}{l}\text { Death due to other } \\
\text { cause }\end{array}$ & 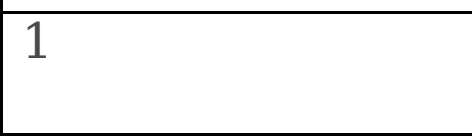 & 2 \\
\hline \multicolumn{3}{|c|}{ Characteristics of COVID-19 in doctors $(n=404)$} \\
\hline & $\begin{array}{l}\text { Doctors who } \\
\text { received single } \\
\text { dose, } n=27\end{array}$ & $\begin{array}{l}\text { Doctors who received } \\
\text { both doses, } n=377\end{array}$ \\
\hline $\begin{array}{l}\text { Confirmed or suspect } \\
\text { cases, n (\%) }\end{array}$ & $19(70.4)$ & $\begin{array}{l}160 \text { (42.4) } \\
\leq 14 \text { days after second dose: } \\
\mathrm{n}=12 \\
>14 \text { days after second dose: } \\
\mathrm{n}=148\end{array}$ \\
\hline $\begin{array}{l}\text { RT-PCR confirmed } \\
\text { SARS-CoV-2 infections } \\
\text { COVID-19 suspect (Not } \\
\text { tested)* } \\
\text { RT-PCR negative } \\
\text { COVID-19 suspect }\end{array}$ & $\begin{array}{l}18(66.7) \\
0(0) \\
1(3.7)\end{array}$ & $\begin{array}{l}131(34.7) \\
17(4.5) \\
12(3.2)\end{array}$ \\
\hline $\begin{array}{l}\text { Severity (all confirmed } \\
\text { or suspect) } \\
\text { Asymptomatic } \\
\text { Mild } \\
\text { Moderate } \\
\text { Severe }\end{array}$ & $\begin{array}{l}1(5.3) \\
14(73.7) \\
3(15.8) \\
1(5.3)\end{array}$ & $\begin{array}{l}9(5.6) \\
130(81.3) \\
16(10) \\
5(3.1)\end{array}$ \\
\hline Hospitalization needed & 1 & $\begin{array}{l}13 \text { (5 for oxygen } \\
\text { requirement, } 7 \text { for } \\
\text { observation, } 1 \text { for general } \\
\text { caregiver support) }\end{array}$ \\
\hline $\begin{array}{l}\text { Death due to other } \\
\text { cause }\end{array}$ & 0 & 1 \\
\hline
\end{tabular}


Table 1. Demographic characteristics and details of COVID-19 in vaccinated individuals

[COVID-19 suspect cases are those in whom the investigators suspected COVID-19 based on clinical pattern of symptoms, exposure to confirmed/probable cases of COVID-19 within the past 14 days, and general guidance from the clinical guidelines issued by the Ministry of Health and Family Welfare (MoHFW), Government of India. They were divided into:

*COVID-19 suspect (Not tested)- in the absence of rapid antigen test or RTPCR test

\#RT-PCR negative COVID-19 suspect- with a negative RT-PCR test

"As defined by the MoHFW, Government of India (For severity, all percentages are out of total confirmed and suspect infections)

Abbreviations- ASD: atrial septal defect, CAD: coronary artery disease, RHD: rheumatic heart disease, RT-PCR: reverse transcriptase- polymerase chain reaction] 